Article

\title{
Comprehensive Evaluation of Power Quality Based on an Improved TOPSIS Method Considering the Correlation between Indices
}

\author{
Youhua Jiang ${ }^{1}$, Mingshuo Fang ${ }^{1, *}$, Ziqi Liu ${ }^{1}$ and Wenji Wang ${ }^{2}$ \\ 1 School of Electronics and Information Engineering, Shanghai University of Electric Power, \\ Shanghai 200090, China \\ 2 State Grid Hubei Electric Power Company, Wuhan 430013, China \\ * Correspondence: ms_shiep@126.com
}

Received: 16 July 2019; Accepted: 29 August 2019; Published: 2 September 2019

\begin{abstract}
In order to improve the scientific and rationality of power quality (PQ) comprehensive evaluation, an improved Technique for Order Preference by Similarity to an Ideal Solution (TOPSIS) strategy in consideration of the correlation between indices is proposed to evaluate PQ. The strategy overcomes the shortcomings of the traditional methods that ignore the correlation between PQ performance parameters. Firstly, the AHP-entropy weight (EW) method is obtained by combining the improved analytic hierarchy process (AHP) and the EW method, and the combined weights of the PQ indices are calculated. Secondly, the Mahalanobis distance is used to replace the Euclidean distance in the traditional TOPSIS method, and the PQ samples that need to be evaluated are sorted. The Mahalanobis distance nonlinearly correlates the components inside the evaluation matrix through its own covariance matrix, which solves the problem that the dimensions of each index are not uniform and eliminates the correlation interference between the indices. The example shows that the improved TOPSIS method effectively avoids the misjudgment caused by the correlation between the indices, and the evaluation results are more reasonable and scientific, with greater superiority and effectiveness.
\end{abstract}

Keywords: power quality; comprehensive evaluation; correlation between indices; improved TOPSIS method; entropy weight method; improved analytic hierarchy process; Mahalanobis distance

\section{Introduction}

The gradual depletion of fossil fuels has not only led to tight energy supplies, but has also caused serious environmental problems [1,2]. This has driven the global dependence on renewable energy. In addition, due to the fragility of the large power grid, a series of large power outages have occurred, and the distributed power generation system can improve the stability of the system to some extent. Therefore, these factors together lead to an increasing penetration of distributed generation in the power system. However, the distributed generation (DG) grid-connecting process generally requires a large number of power electronic interface devices for energy conversion [3], so the grid connection will have a potential impact on power quality (PQ). It may cause problems such as frequency deviation, voltage fluctuation, voltage flicker, voltage unbalance, harmonic distortion, and DC injection $[4,5]$. As DG systems account for a larger proportion of power system capacity, DG has an increasingly greater impact on the PQ of distribution networks. Therefore, establishing a comprehensive PQ comprehensive evaluation model can not only accurately know the operation level of each DG system, but also provide a basis for DG grid-connected pricing, which is of great significance. 
Comprehensive evaluation of PQ is an important approach to quantify the overall PQ performance, and its results can be used as an important reference to distinguish PQ. The overall performance of $\mathrm{PQ}$ is usually derived from a comprehensive description of multiple indices [6]. In order to obtain more comprehensive and perfect power performance, it is necessary to comprehensively evaluate and analyze the PQ data, and its core task is to obtain a single quantitative index by means of weighting and merging of multiple PQ indices [7,8] — that is, to sort the PQ samples that contain multiple power quality evaluation indices.

However, the current PQ evaluation methods have not considered the correlation between the PQ indices, or there are some shortcomings such as the weighting methods being simple, a large amount of calculation, and a lack of practical engineering guidance [9-11]. The artificial neural network method proposed in [12] requires a large amount of sample data to train the network. The fuzzy comprehensive evaluation method proposed in [13-15] relies too much on experience and is too subjective in the process of determining membership function and obtaining weights. The traditional analytic hierarchy process (AHP) proposed in $[16,17]$ needs to construct a judgment matrix, and the calculation amount is too large. The entropy weight (EW) method and its combination method proposed in $[18,19]$ do not take subjective weights into account, which may result in inaccurate weights. In [20], a TOPSIS method based on entropy weight is proposed. The algorithm sorts the PQ samples by calculating the proximity to the ideal solution, where the proximity is calculated by the Euclidean distance between power performance of $\mathrm{PQ}$ sampling points and positive and negative ideal solutions. This method of adopting double reference values avoids the situation of juxtaposed evaluation results.

However, after the correlation analysis of the measured data of $\mathrm{PQ}$, it is found that each batch of indices participating in the evaluation shows a high correlation, which has not received attention in the previous PQ evaluation methods. If the Euclidean distance in the traditional TOPSIS method is replaced by the Mahalanobis distance, the correlation between the PQ indices can be considered, and the juxtaposition of the evaluation results can be avoided, making the evaluation results more scientific.

Therefore, this paper proposes an improved TOPSIS method for comprehensive evaluation of power quality considering the correlation of indices. The distance algorithm in the traditional TOPSIS method is improved, and Mahalanobis distance is adopted to replace Euclidean distance, which can avoid the juxtaposition of evaluation results and effectively overcome the misjudgment caused by the correlation between PQ indices. Due to the weighting being the precondition of PQ evaluation, this paper also proposes a new AHP-EW weighting method based on the combination of improved analytic hierarchy process (AHP) and entropy weight (EW) method to calculate the subjective and objective weights of indices. Compared with the traditional subjective and objective weighting methods, this method can consider the subjective and objective factors at the same time, and greatly reduce the amount of calculation, with strong operability. Finally, the statistical values of PQ monitoring data are evaluated by an example, and the results are compared with the traditional methods to verify the validity and correctness of the proposed algorithm.

Figure 1 shows the improved process of the TOPSIS method. It can be seen that the EW-AHP is used to replace the existing single weighting method, and the objective weight of the original data is obtained by EW. The subjective weight can be obtained by AHP. The combination of the two can take into account the subjective and objective weights at the same time. The Mahalanobis distance replaces the Euclidean distance and eliminates the impact of the correlation between the evaluation indices on the evaluation results. 


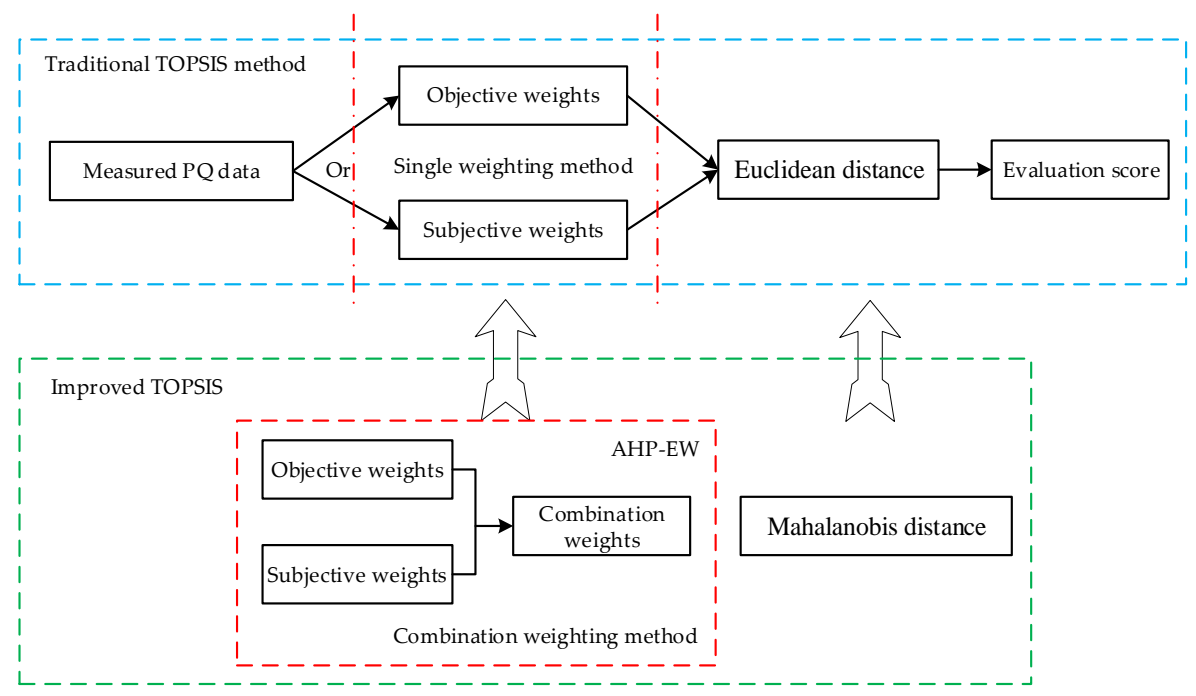

Figure 1. Improved process of the Technique for Order Preference by Similarity to an Ideal Solution (TOPSIS) method.

It should be noted that the evaluation method proposed in this paper is universal. It can be widely used in the evaluation of multiple targets with multiple indices, as shown in Figure 2, including energy efficiency evaluation, service satisfaction evaluation, risk evaluation and so on. Next, the paper will apply the proposed method to PQ evaluation to verify its effectiveness.

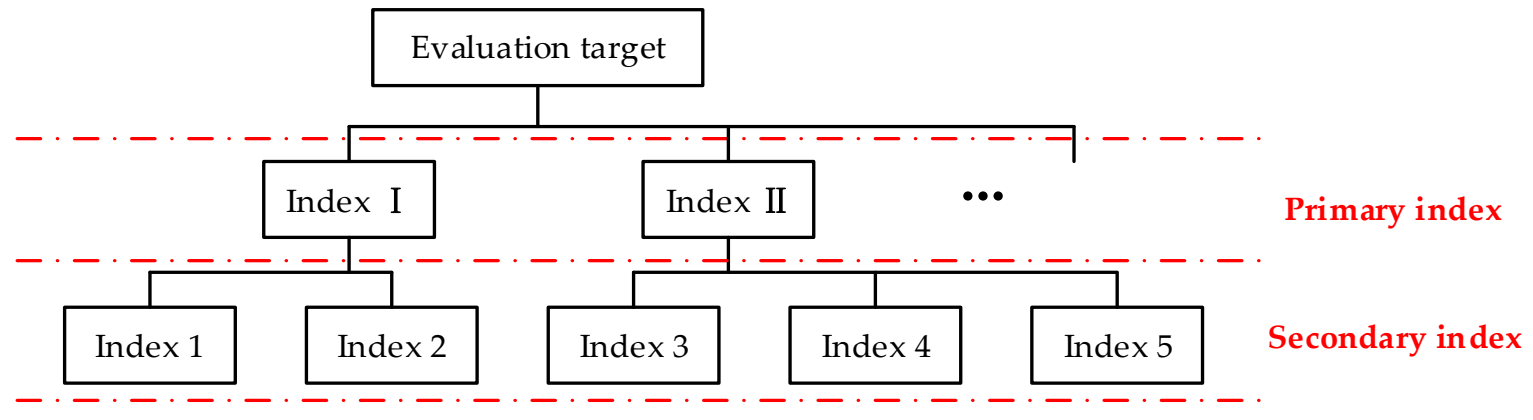

Figure 2. Evaluation system diagram.

\section{Improved TOPSIS Method}

As a common multi-objective decision analysis method [21,22], TOPSIS sorts the evaluation samples based on their proximity to the ideal effect. The algorithm constructs the virtual best solution and the worst solution, namely the positive ideal solution and the negative ideal solution, and calculates the relative Euclidean distance of the evaluation sample and the positive and negative ideal solutions, which is close to the positive ideal solution and far from the negative ideal solution as the optimal solution, and then sorts the evaluation samples in order.

The positive ideal solution and the negative ideal solution are the ideal values of the hypothesis, that is, the positive ideal solution is the virtual best feasible solution, and the negative ideal solution is the virtual worst feasible solution. Figure 3 is a schematic diagram of the decision of the TOPSIS method for two objectives. In the figure, $\mathrm{A}^{+}$and $\mathrm{A}^{-}$are positive ideal solutions and negative ideal solutions, respectively, which are virtual optimal solutions and worst solutions. Although $A_{1}$ is closer to the positive ideal solution than $A_{2}$, it is not far from the negative ideal solution. It can be seen that $\mathrm{A}_{2}$ is farther away from the negative ideal solution. Therefore, the advantage of TOPSIS method is that, among the solutions with the same distance from the positive ideal solution, the one with a longer distance from the negative ideal solution is selected as the satisfactory solution, which avoids the situation where a single ideal solution results in the same evaluation result. 


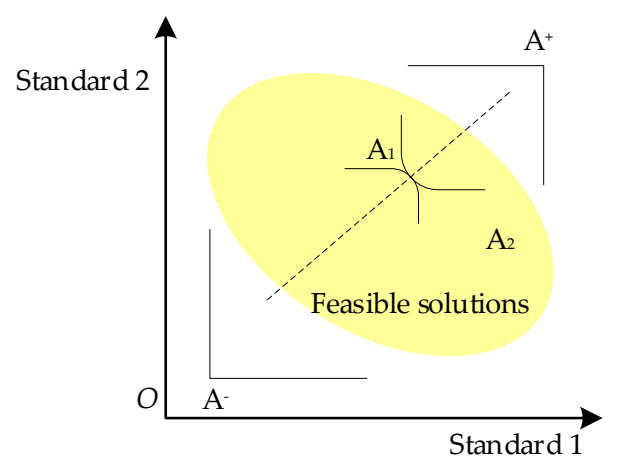

Figure 3. Decision diagram of the TOPSIS method.

Some researchers have improved the TOPSIS method by adding weights again in the calculation process of Euclidean distance. However, the above improvements are only supplemented by the weight issue, and the correlation between PQ indices is still not considered. If the correlation between the indices is neglected, the evaluation results obtained by traditional PQ evaluation methods will inevitably have deviations. In this paper, the Euclidean distance in the traditional TOPSIS method is replaced by the Mahalanobis distance. The Mahalanobis distance nonlinearly correlates the internal components of the evaluation matrix by its own covariance matrix. It can not only solve the problem of different dimensions of indices, but also eliminate the correlation interference between indices. It should be one of the preferred solutions for multi-objective evaluation.

\section{Determine the Weights of PQ Indices}

The weighting method combining subjectivity and objectivity, which can not only consider experts' opinions, but also take into account the impact of indices' data changes on evaluation results, has been applied in the fields of environment, medicine, computer, machinery, etc. [23-26]. In this paper, the improved AHP and EW method are combined to form a new combined weight calculation method called AHP-EW. The improved AHP eliminates the need to construct the judgment matrix, avoids the consistency test, and greatly reduces the computation.

\subsection{Determination of Subjective Weight Based on Improved AHP}

AHP is a multi-objective decision-making method founded by American operations researcher T. L. Satty. Its core idea is to rank the evaluation indices according to the experience of experts $[27,28]$. However, the traditional AHP usually constructs a judgment matrix and needs to pass the consistency test. In the actual calculation process, the test is often difficult to pass. As shown in Figure 4a, the portion of the red dashed box is uncontrollable. Therefore, the amount of calculation of the conventional AHP is large. Moreover, when the order of the judgment matrix is high, the amount of calculation will be larger. Therefore, experts [29] proposed using the scale method to construct the judgment matrix, as shown in Figure $4 \mathrm{~b}$. The improved AHP avoids the consistent test by constructing a judgment matrix, making the calculation process more straightforward and reducing the workload greatly. 

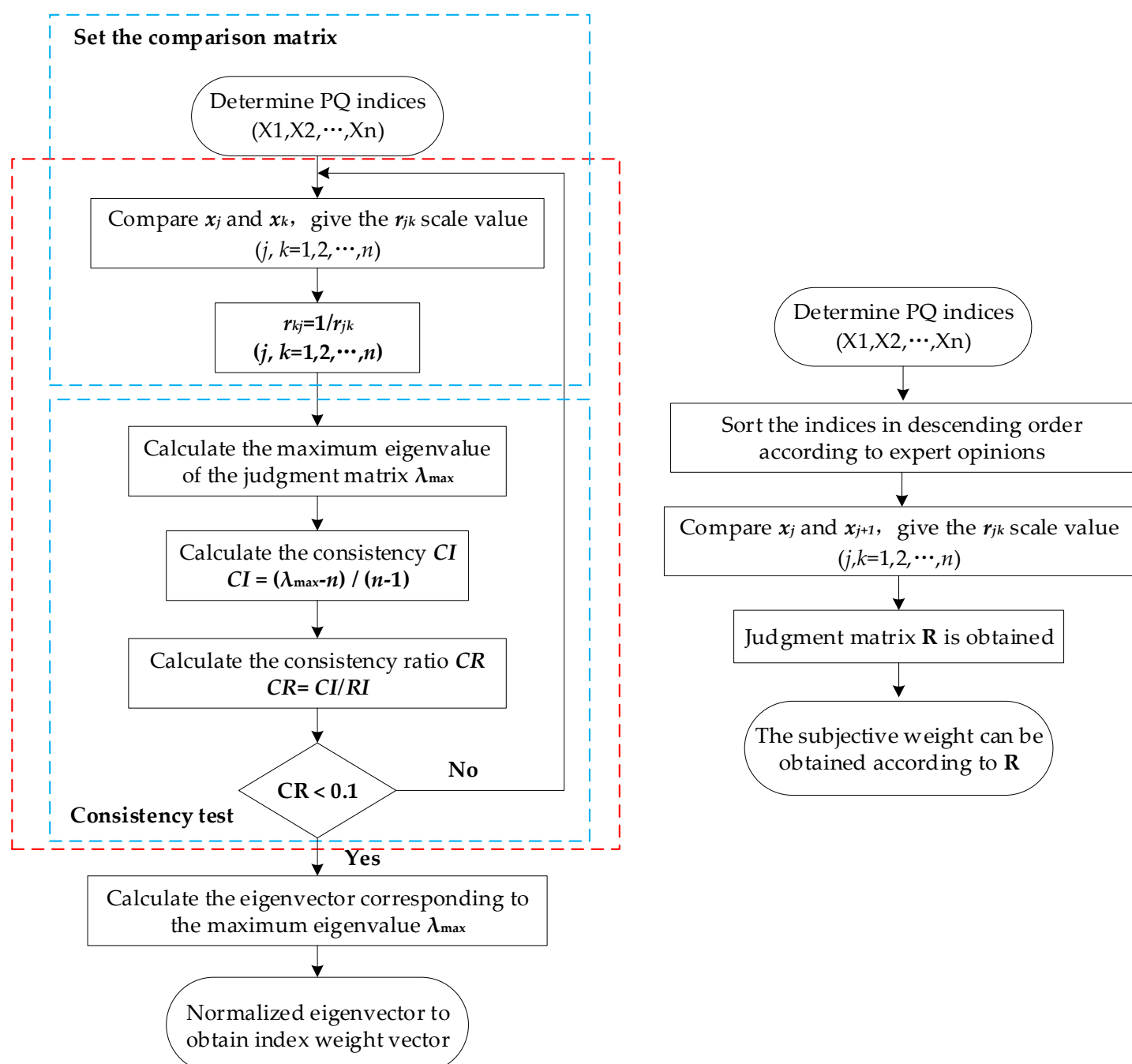

(a)

(b)

Figure 4. Comparison between traditional AHP and improved AHP: (a) flow chart of traditional AHP; (b) flow chart of improved AHP.

Therefore, this paper uses the comparison scale to construct the judgment matrix. It is assumed that an evaluation sample has $n$ indices, according to the expert opinion, the $n$ indices are sorted in descending order of importance, such as: index $1>$ index $2>\ldots>$ index $n$. By comparing the index $j$ and the index $j+1$ in pairs, the corresponding scale is recorded as $b_{j}$. The specific definition of the scale is shown in Table 1.

Table 1. The meanings of scale values.

\begin{tabular}{cc}
\hline Scale Values $\boldsymbol{b}_{j}$ & Scale Definition \\
\hline 1 & A and B are equally important \\
1.2 & A is slightly more important than B \\
1.4 & A is obviously more important than B \\
1.6 & A is strongly more important than B \\
1.8 & A is extremely more important than B \\
$1.1,1.3,1.5,1.7$ & Intermediate values of the above-mentioned judgment. \\
\hline
\end{tabular}

According to the scale assignment method described above, the judgment matrix $R=\left[r_{j k}\right]$ is constructed, and the matrix is defined as follows: 
$r_{j k}$ is the scale value after comparing the $j$ th index with the $k$ th index, where $j, k=1,2, \ldots, n$; $r_{k j}=1 / r_{j k}$, where $j, k=1,2, \ldots, n$;

$r_{j j}=1$, where $j=1,2, \ldots, n$.

Based on the above definition, the judgment matrix $R=\left[r_{j k}\right]$ can be obtained as follows:

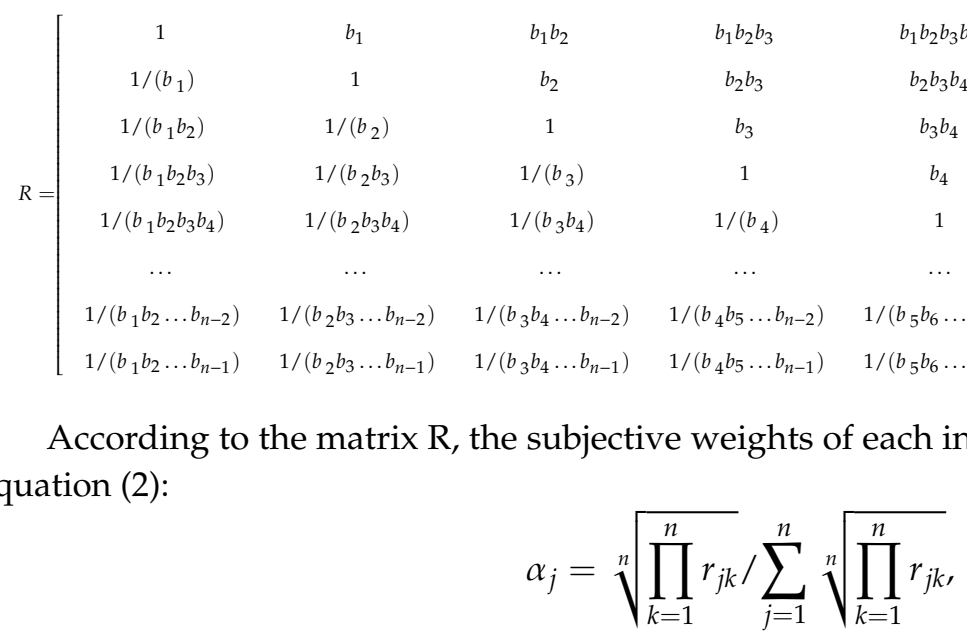$$
\left.\begin{array}{ccc}
b_{1} b_{2} b_{3} b_{4} b_{5} & \ldots & b_{1} b_{2} \ldots b_{n-1} \\
b_{2} b_{3} b_{4} b_{5} & \ldots & b_{2} b_{3} \ldots b_{n-1} \\
b_{3} b_{4} b_{5} & \ldots & b_{3} b_{4} \ldots b_{n-1} \\
b_{4} b_{5} & \ldots & b_{4} b_{5} \ldots b_{n-1} \\
b_{5} & \ldots & b_{5} b_{6} \ldots b_{n-1} \\
\ldots & \ldots & \ldots \\
1 & \ldots & b_{n-1} \\
1 /\left(b_{6} \ldots b_{n-1}\right) & \ldots & 1
\end{array}\right]
$$

where $\alpha_{j}$ is the subjective weight of each index, $j, k=1,2, \ldots, n$.

\subsection{Determination of Objective Weight Based on the EW Method}

C. E. Shannon, the founder of information theory, introduced entropy theory into information theory as a measure of information disorder $[30,31]$. In this paper, the EW method is used to determine the objective weight of each index of PQ. As an objective method of weighting, the entropy method can obtain objective weights independently of subjective factors. The smaller the entropy value, the lower the information disorder, and the higher the available value, so the corresponding index weight should be larger. On the contrary, the larger the entropy value, the higher the information disorder, and the lower the available value, so the corresponding index weight should be smaller. The detailed relationship is shown in Table 2.

Table 2. Relationship between entropy weight and entropy value.

\begin{tabular}{cccc}
\hline & Disorder of Information & Value of Information & Weight to Be Given \\
\hline Large entropy value & High & Low & Small \\
Small entropy value & Low & High & Large \\
\hline
\end{tabular}

Let the evaluation matrix be $A=\left(a_{i j}\right)_{\mathrm{m} * \mathrm{n}}$, and the objective weights calculation procedure of the indices is as follows [32]:

Standardization processing of evaluation data using standard 0-1 transformation:

When the index is a benefit attribute index, that is, the larger the index value, the better. The standardization equation is as follows:

$$
a_{i j}^{*}=\frac{a_{i j}-\operatorname{mina}_{j}}{\operatorname{maxa}_{j}-\operatorname{mina}_{j}}, i=1,2, \ldots, m ; j=1,2, \ldots, n .
$$


When the index is a cost-based attribute index, that is, the smaller the index value, the better. The standardization equation is as follows:

$$
a_{i j}^{*}=\frac{\operatorname{maxa}_{i j}-a_{i j}}{\max _{j} a_{i j}-\operatorname{mina}_{i j}}, i=1,2, \ldots, m ; j=1,2, \ldots, n,
$$

where $b_{i j}$ is the standardized value of the $j$ th index of the $i$ th evaluation sample.

1 According to the standardized decision matrix, the characteristic proportion of the $j$ th index of the $i$ th evaluation sample:

$$
p_{i j}=a_{i j}^{*} / \sum_{i=1}^{m} a_{i j}^{*}, j=1,2, \ldots, n
$$

where, $0<p_{i j}<1$.

2 The entropy value of the $j$ th index is:

$$
e_{j}=-\frac{1}{\ln (m)} \sum_{i=1}^{m} p_{i j} \ln \left(p_{i j}\right), j=1,2, \ldots, n,
$$

where $p_{i j} \ln \left(p_{i j}\right) \rightarrow 0$ for $p_{i j} \rightarrow 0$.

3 Difference coefficient of the $j$ th index can be obtained as follows:

$$
g_{j}=1-e_{j}, j=1,2, \ldots, n
$$

4 The entropy weight $\beta_{j}$ of the $j$ th index can be obtained as follows:

$$
\beta_{j}=g_{j} / \sum_{j=1}^{n} g_{j}, j=1,2, \ldots, n
$$

According to Figure 5, we can understand the relationship between entropy value and entropy weight intuitively, and how the objective weight is obtained from the original data.

\begin{tabular}{|c|c|c|c|c|c|}
\hline & Index 1 & Index 2 & Index 3 & $\ldots$ & Index n \\
\hline 1 & $a_{11}$ & $a_{12}$ & $a_{13}$ & $\ldots$ & $a_{1 n}$ \\
\hline 2 & $a_{21}$ & $a_{22}$ & $a_{23}$ & $\ldots$ & $a_{2 n}$ \\
\hline$\ldots$ & $\ldots$ & $\ldots$ & $\ldots$ & $\ldots$ & $\ldots$ \\
\hline$m$ & $a_{m 1}$ & $a_{m 2}$ & $a_{m 3}$ & $\ldots$ & $a_{m n}$ \\
\hline
\end{tabular}

Original information

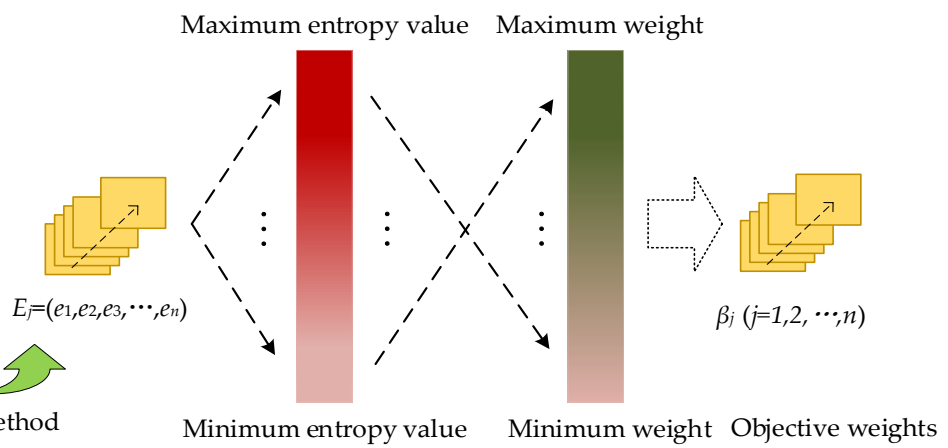

Figure 5. Schematic diagram of obtaining objective weight based on an entropy weight (EW) method.

\subsection{Determination of Combination Weight}

Equation (2) obtains the subjective weights of PQ indices, and Equation (8) obtains the objective weights of PQ indices. The subjective and objective weights of each index are multiplied and normalized, and the combined weight can be obtained as follows:

$$
w_{j}=\left(\alpha_{j} \beta_{j}\right) / \sum_{k=1}^{n} \alpha_{k} \beta_{k}, j=1,2, \ldots, n
$$


It can be seen from the combination weight Equation (9) that it not only considers expert opinions to scale the importance of indices, but also distinguishes the quality of the index data, overcomes the shortcomings of the single weighting method, and meets the engineering practice of PQ evaluation.

\section{Realization of PQ Comprehensive Evaluation}

1. Solve the weighted normalized decision matrix:

Let $\omega_{j}=\left[w_{1}, w_{2}, \ldots, w_{n}\right]^{\mathrm{T}}$ be the combined weight vector of each PQ index, then the weighted normalized decision matrix $C=\left(c_{i j}\right)_{\mathrm{m}^{* \mathrm{n}}}$ can be obtained as follows:

$$
c_{i j}=w_{j} a_{i j}, \quad i=1,2, \ldots, m ; j=1,2, \ldots, n .
$$

2. Determine the absolute ideal solution of the evaluation system:

According to Equation (10), the weighted normalized matrix $C=\left(c_{i j}\right)_{m^{*} n}$ is obtained, and, according to the basic principle of the TOPSIS method, two vectors of positive ideal solution $A_{j}{ }^{+}$and negative ideal solution $A_{j}^{-}$are constructed:

$$
\begin{aligned}
& A_{j}^{+}=\left\{\begin{array}{l}
1, j \in T 1 \\
0, j \in T 2
\end{array},\right. \\
& A_{j}^{-}=\left\{\begin{array}{l}
0, j \in T 1 \\
1, j \in T 2
\end{array},\right.
\end{aligned}
$$

where, 1 and 0 respectively represent the maximum and minimum values of the corresponding indices. $T 1$ means the benefit attribute index, that is, the positive index; $T 2$ means the cost-based attribute index, that is, the inverse index.

3. Calculate the Mahalanobis distance:

Let the multi-index vector $x=\left(x_{1}, x_{2}, \ldots, x_{p}\right)$ whose mean vector is $\mu=\left(\mu_{1}, \mu_{2}, \ldots, \mu_{p}\right)$, and $s^{-1}$ be the co-inverse matrix of $x$, then define the Mahalanobis distance equation for $x$ as follows:

$$
M(x)=\sqrt{(x-\mu)^{T} s^{-1}(x-\mu) .}
$$

Combined with Equation (13), the Mahalanobis distance of the $i$ th PQ evaluation sample to the positive and negative ideal solutions can be respectively defined as follows:

$$
\begin{aligned}
\widetilde{M}\left(A_{i}, A^{+}\right) & =\sqrt{\left(A_{i}-A^{+}\right)^{T} s^{-1}\left(A_{i}-A^{+}\right)}, \\
\widetilde{M}\left(A_{i}, A^{-}\right) & =\sqrt{\left(A_{i}-A^{-}\right)^{T} s^{-1}\left(A_{i}-A^{-}\right)} .
\end{aligned}
$$

4. Calculate the proximity of the $i$ th evaluation sample:

Let $c_{i}$ be the relative proximity of the $i$ th evaluation index to the ideal solution, and sort the evaluation sample according to the values of proximity $c_{i}$. The larger the $c_{i}$, the better the evaluation sample. Conversely, the smaller the $c_{i}$, the worse the evaluation sample. The $c_{i}$ can be obtained as follows:

$$
c_{i}=\frac{\widetilde{M}\left(A_{i}, A^{-}\right)}{\widetilde{M}\left(A_{i}, A^{-}\right)+\widetilde{M}\left(A_{i}, A^{+}\right)}, i=1,2, \ldots, m .
$$

5. An improved TOPSIS method for comprehensive evaluation of PQ considering the correlation between indices is shown in Figure 6: 


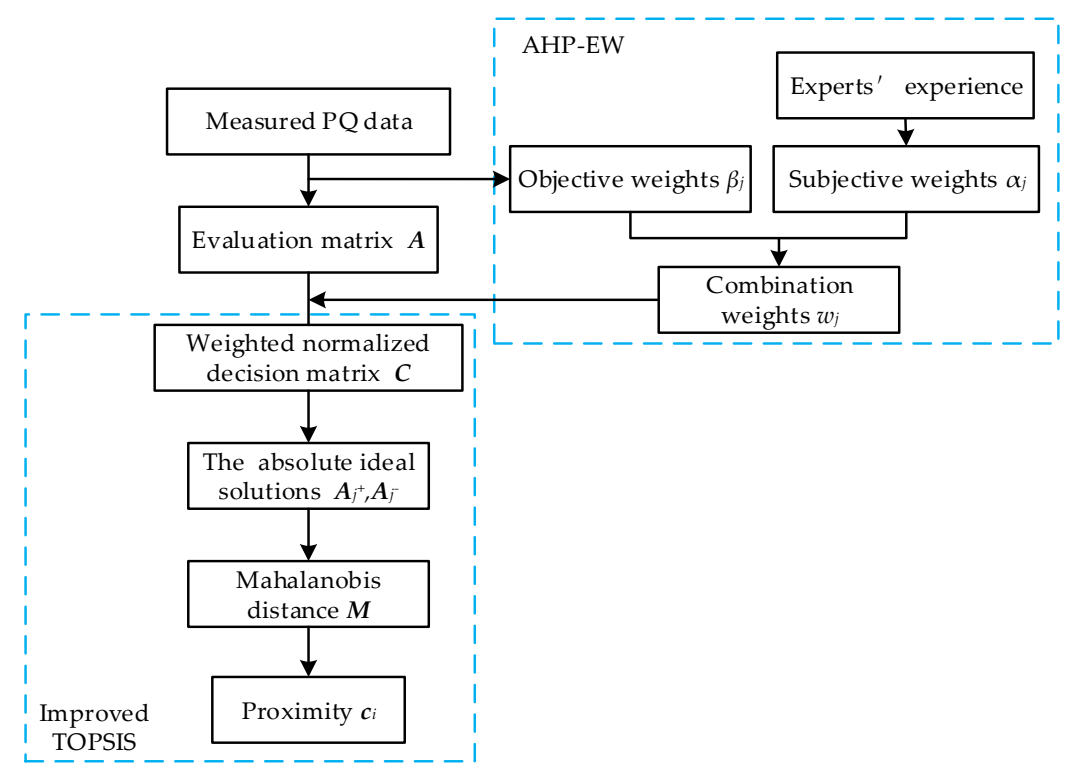

Figure 6. The flowchart of power quality (PQ) comprehensive evaluation based on the improved TOPSIS method.

\section{Experimental Results}

The PQ performance is usually determined in terms of six indices, namely frequency deviation, voltage total harmonic distortion, voltage fluctuation, voltage flicker, voltage deviation and three-phase voltage unbalance. Table 3 shows the measured PQ data of the five major substation busbar nodes of a large wind farm in China, and obtained five sets of PQ indices data sets. The five substation nodes are: $10 \mathrm{kV}$ busbar of substation 1, recorded as sample $1 ; 110 \mathrm{kV}$ busbar of substation 2, recorded as sample 2; $10 \mathrm{kV}$ busbar of substation 3, recorded as sample 3; $35 \mathrm{kV}$ busbar of substation 4, recorded as sample $4 ; 10 \mathrm{kV}$ busbar of substation 5 , recorded as sample 5 .

Take the PQ data of the above five evaluation samples as an example. Since the PQ is fluctuating, the PQ data obtained only once is not sufficient to explain the overall performance of the power. Therefore, in order to obtain reasonable PQ data, long-term monitoring of observation points is required. In the PQ monitoring system, when the PQ data are collected and summarized daily, weekly, monthly, seasonally, and annually, the $95 \%$ probability value is often used as the quantitative statistical index. The data in Table 3 is the statistical value measured in days, which can represent a dynamic PQ level.

Table 3. Measured PQ data.

\begin{tabular}{ccccccc}
\hline Samples/Indices $^{\mathbf{1}}$ & $\boldsymbol{x}_{\mathbf{1}} / \mathbf{H z}$ & $\boldsymbol{x}_{\mathbf{2}} / \%$ & $x_{3} / \%$ & $x_{\mathbf{4}} / \%$ & $x_{5} / \%$ & $x_{\mathbf{6}} / \%$ \\
\hline 1 & 0.09 & 1.12 & 0.96 & 0.22 & 2.53 & 0.88 \\
2 & 0.04 & 1.26 & 1.05 & 0.34 & 1.66 & 1.07 \\
3 & 0.19 & 1.18 & 1.41 & 0.47 & 3.85 & 0.83 \\
4 & 0.11 & 0.82 & 0.85 & 0.38 & 2.01 & 0.58 \\
5 & 0.07 & 1.35 & 1.27 & 0.53 & 3.18 & 1.23 \\
\hline
\end{tabular}

1 - $x_{1}$-frequency deviation, $x_{2}$-voltage total harmonic distortion, $x_{3}$-voltage fluctuation, $x_{4}$-voltage flicker, $x_{5}$-voltage deviation, $x_{6}$-three-phase voltage unbalance.

The original data in Table 3 was imported into the software SPSS (version, Manufacturer, City, US State abbrev. if applicable, Country) for correlation analysis, and the Pearson correlation coefficient (Pcc) was obtained, as shown in Table 4. It can be seen from Table 4 that, under the two-sided test of the significance level of 0.01 , the correlation coefficient between the voltage deviation and the three-phase voltage unbalance is 1 , and the probability value of the two-sided test is 0 , less than 0.01 , which means the correlation degree is significant and completely positive. Under the two-sided test of 
the significance level of 0.05 , the correlation coefficient between the voltage deviation and the voltage total harmonic distortion is 0.95 , and the probability of the two-sided test is 0.013 , less than 0.05 , which means that the correlation degree is significant and highly positive. The correlation coefficient between voltage total harmonic distortion and three-phase voltage unbalance is 0.95 , and the probability of two-sided test is 0.013 , less than 0.05 , which means that the correlation degree is significant and highly positive. Therefore, there is a clear correlation between some indices. In this case, if traditional evaluation methods are still adopted, certain errors will be caused.

Table 4. Pearson correlation analysis of measured data.

\begin{tabular}{cccccccc}
\hline & & $x_{\mathbf{1}}$ & $x_{\mathbf{2}}$ & $x_{\mathbf{3}}$ & $x_{\mathbf{4}}$ & $x_{\mathbf{5}}$ & $x_{\mathbf{6}}$ \\
\hline \multirow{2}{*}{$x_{\mathbf{1}}$} & Pcc & 1 & -0.526 & 0.477 & 0.280 & -0.283 & -0.526 \\
& significance level & - & 0.362 & 0.416 & 0.648 & 0.645 & 0.362 \\
\hline \multirow{2}{*}{$x_{\mathbf{2}}$} & Pcc & -0.526 & 1 & 0.478 & 0.328 & $0.950^{1}$ & $1.000^{2}$ \\
& significance level & 0.362 & - & 0.415 & 0.590 & 0.013 & 0.000 \\
\hline \multirow{2}{*}{$x_{\mathbf{3}}$} & Pcc & 0.477 & 0.478 & 1 & 0.706 & 0.676 & 0.478 \\
& significance level & 0.416 & 0.415 & - & 0.182 & 0.211 & 0.415 \\
\hline \multirow{2}{*}{$x_{\mathbf{4}}$} & Pcc & 0.280 & 0.328 & 0.706 & 1 & 0.344 & 0.328 \\
& significance level & 0.648 & 0.590 & 0.182 & - & 0.571 & 0.590 \\
\hline \multirow{2}{*}{$x_{5}$} & Pcc & -0.283 & $0.950^{1}$ & 0.676 & 0.344 & 1 & $0.950^{1}$ \\
& significance level & 0.645 & 0.013 & 0.211 & 0.571 & - & 0.013 \\
\hline \multirow{2}{*}{$x_{\mathbf{6}}$} & Pcc & -0.526 & $1.000^{2}$ & 0.478 & 0.328 & $0.950^{1}$ & 1 \\
& significance level & 0.362 & 0.000 & 0.415 & 0.590 & 0.013 & - \\
\hline
\end{tabular}

${ }^{1}$ Correlation is significant on the 0.05 layer (two sides), ${ }^{2}$ Correlation is significant on the 0.01 layer (two sides).

The PQ measured data of Table 3 will be comprehensively evaluated according to the method proposed in this paper. The first step is to determine the combined weights of the six PQ indices.

The AHP is used to calculate the subjective weights of the indices. According to the experts' opinions, the six indices can be arranged in descending order of importance as follows: frequency deviation $>$ voltage total harmonic distortion $>$ voltage fluctuation $\geq$ voltage flicker $>$ voltage deviation $>$ three-phase voltage unbalance. The scale values can be determined according to the experts' opinions when it is actually applied. Here, it is assumed that $r_{12}=1.8, r_{23}=1.7, r_{34}=1, r_{45}=1.4, r_{56}=1.2$. According to the Equation (1), the judgment matrix $\boldsymbol{R}$ can be calculated as follows:

$$
R=\left[\begin{array}{cccccc}
1 & 1.8 & 3.06 & 3.06 & 4.284 & 5.1408 \\
1 / 1.8 & 1 & 1.7 & 1.7 & 2.38 & 2.856 \\
1 / 3.06 & 1 / 1.7 & 1 & 1 & 1.4 & 1.68 \\
1 / 3.06 & 1 / 1.7 & 1 & 1 & 1.4 & 1.68 \\
1 / 4.284 & 1 / 2.38 & 1 / 1.4 & 1 / 1.4 & 1 & 1.2 \\
1 / 5.1408 & 1 / 2.856 & 1 / 1.68 & 1 / 1.68 & 1 / 1.2 & 1
\end{array}\right] .
$$

According to Equation (2), the subjective weights of six indices such as frequency deviation, voltage total harmonic distortion, voltage fluctuation, voltage flicker, voltage deviation and three-phase voltage unbalance are calculated in order:

$$
\boldsymbol{\alpha}_{j}=(0.379205,0.210669,0.123923,0.123923,0.088516,0.073764) \text {. }
$$

The EW method is used to calculate the objective weights of the indices. The data can be normalized according to Equations (4) and (5) to obtain the characteristic proportion matrix $\boldsymbol{P}_{i j}$. The objective weights of each index can be calculated according to Equations (6)-(8):

$$
\boldsymbol{\beta}_{j}=(0.126304,0.212929,0.161242,0.18105,0.153006,0.165469) .
$$


According to Equation (9), the combined weights of PQ indices can be calculated as follows:

$$
w_{j}=(0.297634,0.278758,0.124171,0.139425,0.084163,0.075849) .
$$

According to Equation (10), the measured data of Table 3 is weighted to obtain a weighted normalized matrix $C_{i j}$, as shown in Table 5. In addition, the positive and negative ideal solutions are determined by Table 5, which are:

$$
\begin{aligned}
& A^{+}=(0.011905,0.228581,0.105545,0.030674,0.139711,0.043992), \\
& A^{-}=(0.056550,0.376323,0.175081,0.073895,0.324028,0.093294) .
\end{aligned}
$$

Table 5. Weighted normalized matrix.

\begin{tabular}{ccccccc}
\hline Samples/Indices & $\boldsymbol{x}_{\mathbf{1}}$ & $\boldsymbol{x}_{\mathbf{2}}$ & $\boldsymbol{x}_{\mathbf{3}}$ & $\boldsymbol{x}_{\mathbf{4}}$ & $\boldsymbol{x}_{\mathbf{5}}$ & $\boldsymbol{x}_{\mathbf{6}}$ \\
\hline 1 & 0.026787 & 0.312209 & 0.119204 & 0.030674 & 0.212933 & 0.066747 \\
2 & 0.011905 & 0.351235 & 0.130379 & 0.047405 & 0.139711 & 0.081158 \\
3 & 0.056550 & 0.328934 & 0.175081 & 0.065530 & 0.324028 & 0.062955 \\
4 & 0.032740 & 0.228581 & 0.105545 & 0.052982 & 0.169168 & 0.043992 \\
5 & 0.020834 & 0.376323 & 0.157697 & 0.073895 & 0.267639 & 0.093294 \\
\hline
\end{tabular}

According to Equations (14) and (15), the Mahalanobis distance of each evaluation sample to positive and negative ideal solutions can be obtained by Matlab (version, Manufacturer, City, US State abbrev. if applicable, Country):

$$
\begin{aligned}
& M_{i}{ }^{+}=(2.1176,2.5083,2.9249,1.0889,3.1036), \\
& M_{i}^{-}=(2.6738,2.6294,1.2683,2.9900,1.7596) .
\end{aligned}
$$

According to Equation (16), the proximity of each evaluation sample can be obtained, which are: $c_{1}=0.558041, c_{2}=0.511785, c_{3}=0.302466, c_{4}=0.733041, c_{5}=0.361819$. Then, the proximity of each evaluation sample is sorted as: $c_{4}>c_{1}>c_{2}>c_{5}>c_{3}$.

In order to test the superiority and rationality of the proposed algorithm, the calculation results of the traditional TOPSIS method in [15] are compared with the results in this paper, as shown in Table 6. It can be seen that the proximity values calculated by the traditional TOPSIS method in [15] are: $c_{4}>c_{1}$ $>c_{2}>c_{3}>c_{5}$, that is, sample $4>$ sample $1>$ sample $2>$ sample $3>$ sample 5 , where, " $>$ " means "better than". The proximity values calculated by the method in this paper are sorted as: $c_{4}>c_{1}>c_{2}>c_{5}>$ $c_{3}$, that is, sample $4>$ sample $1>$ sample $2>$ sample $5>$ sample 3 . Obviously, the improved TOPSIS method proposed in this paper differs from the traditional TOPSIS method in the ranking of samples 3 and 5 .

Table 6. Comparison of evaluation results.

\begin{tabular}{cccccc}
\hline & Sample 1 & Sample 2 & Sample 3 & Sample 4 & Sample 5 \\
\hline Traditional TOPSIS method & 0.492999 & 0.395786 & 0.271828 & 0.858968 & 0.213107 \\
Proposed method & 0.558041 & 0.511785 & 0.302466 & 0.733041 & 0.361819 \\
\hline
\end{tabular}

According to the experience given by experts, the importance of the six PQ indices in descending order is as follows: frequency deviation $>$ voltage total harmonic distortion $>$ voltage fluctuation $>$ voltage flicker $>$ voltage deviation $>$ three-phase voltage unbalance. According to the above order, the PQ of the samples 3 and 5 are compared in detail, as shown in Table 7. It can be seen that the sample 5 is better than the sample 3 , and this result is consistent with the evaluation result of this paper. 
Table 7. Comparison of samples 3 and 5.

\begin{tabular}{ccccc}
\hline & Sample 3 & Sample 5 & Importance & Contrast \\
\hline$x_{1} / \mathrm{Hz}$ & 0.19 & 0.07 & 1 & Sample 5 better \\
$x_{2} / \%$ & 1.18 & 1.35 & 2 & Sample 3 better \\
$x_{3} / \%$ & 1.41 & 1.27 & 3 & Sample 5 better \\
$x_{4} / \%$ & 0.47 & 0.53 & 4 & Sample 3 better \\
$x_{5} / \%$ & 3.85 & 3.18 & 5 & Sample 5 better \\
$x_{6} / \%$ & 0.83 & 1.23 & 6 & Sample 3 better \\
\hline
\end{tabular}

In order to further verify the effectiveness of the improved TOPSIS method for comprehensive evaluation of $\mathrm{PQ}$ in consideration of the correlation of indices proposed in this paper, the evaluation results of existing evaluation methods, including AHP, fuzzy theory method, combined weighting method, traditional TOPSIS method, and the results of this paper are compared, as shown in Table 8 . In the process of calculation, the traditional AHP needs to construct the judgment matrix, and the consistency test is performed many times, that is, the calculation amount is huge. The fuzzy theory method is too subjective because it relies too much on experience in determining the membership function and obtaining weights, and does not consider the information of the data itself. The combined weighting method only aggregates the PQ data through the combination weights of the indices, and lacks the rigor of engineering mathematics. None of the above methods took measures to resolve the correlation between PQ indices. It can be seen from Table 8 that the evaluation results of this paper are consistent with the evaluation results of other methods, which is consistent with the fitting trend of each evaluation result, which confirms the effectiveness and superiority of the proposed method.

Table 8. Comparison of results of each evaluation method.

\begin{tabular}{cl}
\hline Evaluation Methods & \multicolumn{1}{c}{ Power Quality Evaluation Results } \\
\hline Analytic hierarchy process & Point $4>$ Point $1>$ Point $2>$ Point $5>$ Point 3 \\
Fuzzy theory method & Point $4>$ Point $2>$ Point $1>$ Point $5>$ Point 3 \\
Combined weight method & Point $1>$ Point $4>$ Point $2>$ Point $5>$ Point 3 \\
Traditional TOPSIS method & Point $4>$ Point $1>$ Point $2>$ Point $3>$ Point 5 \\
Method of this paper & Point $4>$ Point $1>$ Point $2>$ Point $5>$ Point 3 \\
\hline
\end{tabular}

\section{Conclusions}

Aiming at the influence of the correlation between the power quality performance parameters (that is, the PQ indices in this paper) on the comprehensive evaluation results of $P Q$, this paper proposes to establish a comprehensive evaluation model by combining the improved TOPSIS method and the AHP-EW combination weighting method. This method is suitable for a variety of evaluation scenarios, mainly for targets with multiple indices. In these scenarios, you only need to select the required indicators. This paper applies this method to PQ evaluation, through the analysis of actual case data, the following conclusions are drawn:

1. There is a high correlation between some steady state indices of $P Q$, and it has not received attention and treatment in the previous comprehensive evaluation of PQ.

2. The Mahalanobis distance nonlinearly correlates the internal components of the evaluation matrix by its own covariance matrix. It can not only solve the problem of different dimensions of indices, but also eliminate the correlation interference between indices.

3. The new AHP-EW combination weighting method has the advantages of less calculation and strong operability compared with the traditional subjective and objective weighting method;

4. The evaluation results of the proposed method are in line with the fitting trend of other evaluation results, and the evaluation errors of other methods are corrected. The validity and superiority of the proposed TOPSIS method for comprehensive evaluation of PQ can be confirmed. 
5. The correlation between the steady state indices of PQ has been the first concern for the comprehensive evaluation results of $\mathrm{PQ}$, which provides a new reference and consideration for the following work of PQ evaluation.

Author Contributions: All of the authors made contributions to the concept and design of the article. M.F. is the main author of this work; Y.J. provided good advice and technical guidance for the manuscript; Z.L. and W.W. reviewed and polished the manuscript.

Funding: This research was funded by Construction of Local Capacity in Shanghai City, Grant No. 14110500900.

Conflicts of Interest: The authors declare no conflict of interest.

\section{References}

1. Li, Y.; Kao, C. An Accurate Power Control Strategy for Power-Electronics-Interfaced Distributed Generation Units Operating in a Low-Voltage Multibus Microgrid. IEEE Trans. Power Electr. 2009, 24, 2977-2988.

2. He, J.; Li, Y. Analysis, Design, and Implementation of Virtual Impedance for Power Electronics Interfaced Distributed Generation. IEEE Trans. Ind. Appl. 2011, 47, 2525-2538. [CrossRef]

3. Dragicevic, T.; Vazquez, S.; Wheeler, P. Advanced Control Methods for Power Converters in Distributed Generation Systems and Microgrids. IEEE Trans. Ind. Electron. 2019, 66, 8866-8869. [CrossRef]

4. Khadkikar, V. Enhancing Electric Power Quality Using UPQC: A Comprehensive Overview. IEEE Trans. Power Electr. 2011, 27, 2284-2297. [CrossRef]

5. Bojoi, R.; Limongi, L.R.; Roiu, D.; Tenconi, A. Enhanced Power Quality Control Strategy for Single-Phase Inverters in Distributed Generation Systems. IEEE Trans. Power Electr. 2011, 26, 798-806. [CrossRef]

6. Elbasuony, G.S.; Aleem, S.H.E.A.; Ibrahim, A.M.; Sharaf, A.M. A Unified Index for Power Quality Evaluation in Distributed Generation Systems. Energy 2018, 149, 607-622. [CrossRef]

7. Shaik, A.G.; Mahela, O.P. Power Quality Assessment and Event Detection in Hybrid Power System. Electr. Power Syst. Res. 2018, 161, 26-44. [CrossRef]

8. Lu, S.; Wang, L.; Ke, S.; Chang, C.; Yang, Z. Evaluation of Measured Power Quality Results of a Wind Farm Connected to Taiwan Power System. IEEE Trans. Ind. Appl. 2016, 52, 42-49. [CrossRef]

9. Monteiro, L.; Exposto, B.; Pinto, G.; Monteiro, V.; Aredes, M.; Afonso, J.L. Experimental Evaluation of a Control System Based on a Dual-DSP Architecture for a Unified Power Quality Conditioner. Energies 2019, 12, 1694. [CrossRef]

10. Milanovic, J.V.; Abdelrahman, S.; Liao, H.L. Compound index for power quality evaluation and benchmarking. IET Gener. Transm. Dis. 2018, 12, 4269-4275. [CrossRef]

11. Dong, Y.; Liu, Y.; Yin, Z. A Comprehensive Combinatorial Weighting Method for Power Quality Evaluation Based on Maximization Deviation. In Proceedings of the 2nd IEEE Conference on Energy Internet and Energy System Integration (EI2), Beijing, China, 20-22 October 2018.

12. Garanayak, P.; Panda, G. An adaptive linear neural network with least mean M-estimate weight updating rule employed for harmonics identification and power quality monitoring. Trans. Inst. Meas. Control 2018, 40, 1936-1949. [CrossRef]

13. Tian, F.; Jing, Z.; Zhao, H.; Zhang, E.; Liu, J. A Synthetic Condition Assessment Model for Power Transformers Using the Fuzzy Evidence Fusion Method. Energies 2019, 12, 857. [CrossRef]

14. Pau, G.; Collotta, M.; Maniscalco, V. Bluetooth 5 Energy Management through a Fuzzy-PSO Solution for Mobile Devices of Internet of Things. Energies 2017, 10, 992.

15. Yu, H.; Tarsitano, D.; Hu, X.; Cheli, F. Real Time Energy Management Strategy for a Fast Charging Electric Urban Bus Powered by Hybrid Energy Storage System. Energy 2016, 112, 322-331. [CrossRef]

16. Sun, L.; Ma, Z.; Shang, Y.; Liu, Y.; Yuan, H.; Wu, G. Research on Multi-Attribute Decision-Making in Condition Evaluation for Power Transformer Using Fuzzy AHP and Modified Weighted Averaging Combination. IET Gener. Transm. Dis. 2016, 10, 3855-3864. [CrossRef]

17. Zeng, Z.; Yang, H.; Tang, S.; Zhao, R. Objective-Oriented Power Quality Compensation of Multifunctional Grid-Tied Inverters and Its Application in Microgrids. IEEE Trans. Power Electr. 2015, 30, 1255-1265. [CrossRef] 
18. Liang, N.; Li, F.; Guo, Z.; Ji, Y.; Chen, H.; Zheng, H. Research on the Comprehensive Evaluation of Power Quality Based on Customer Side Perspective. In Proceedings of the 2nd International Conference on Power System Technology (POWERCON), Guangzhou, China, 6-8 November 2018.

19. Zhao, H.; Guo, S.; Zhao, H. Comprehensive Performance Assessment on Various Battery Energy Storage Systems. Energies 2018, 11, 2841. [CrossRef]

20. Boran, F.E.; Genç, S.; Kurt, M.; Akay, D. A Multi-Criteria Intuitionistic Fuzzy Group Decision Making for Supplier Selection with TOPSIS Method. Expert Syst. Appl. 2009, 36, 11363-11368. [CrossRef]

21. Lin, C.M.; Huynh, T.T. Function-Link Fuzzy Cerebellar Model Articulation Controller Design for Nonlinear Chaotic Systems Using TOPSIS Multiple Attribute Decision-Making Method. Int. J. Fuzzy Syst. 2018, 20, 1839-1856. [CrossRef]

22. Gligorić, M.; Gligorić, Z.; Beljić, Č.; Torbica, S.; Savić, S.Š.; Ostojić, J.N. Multi-Attribute Technological Modeling of Coal Deposits Based on the Fuzzy TOPSIS and C-Mean Clustering Algorithms. Energies 2016, 9, 1059. [CrossRef]

23. Li, S.; Niu, D.; Wu, L. Evaluation of Energy Saving and Emission Reduction Effects for Electricity Retailers in China Based on Fuzzy Combination Weighting Method. Appl. Sci. 2018, 8, 1564. [CrossRef]

24. Ge, S.; Shi, Z.; Peng, G.; Zhu, Z. Two-Steps Coronary Artery Segmentation Algorithm Based on Improved Level Set Model in Combination with Weighted Shape-Prior Constraints. J. Med. Syst. 2019, 43, 210. [CrossRef] [PubMed]

25. Yu, H.; Sun, X.; Wang, J. Ensemble OS-ELM based on combination weight for data stream classification. Appl. Intell. 2019, 49, 2382-2390. [CrossRef]

26. Gu, Y.; Zhang, M.; Zhou, X. Fault Diagnosis of Gearbox Based on Improved DUCG With Combination Weighting Method. IEEE Access 2019, 7, 92955-92967. [CrossRef]

27. Wang, Y.; Luo, Y.; Hua, Z. On the Extent Analysis Method for Fuzzy AHP and Its Applications. Eur. J. Oper. Res. 2008, 186, 735-747. [CrossRef]

28. Kaur, R.; Singh, S.; Kumar, H. AuthCom: Authorship verification and compromised account detection in online social networks using AHP-TOPSIS embedded profiling based technique. Expert Syst. Appl. 2018, 113, 397-414. [CrossRef]

29. Ishizaka, A.; Labib, A. Review of the Main Developments in the Analytic Hierarchy Process. Expert Syst. Appl. 2011, 38, 14336-14345. [CrossRef]

30. Wu, J.; Sun, J.; Liang, L.; Zha, Y. Determination of Weights for Ultimate Cross Efficiency Using Shannon Entropy. Expert Syst. Appl. 2011, 38, 5162-5165. [CrossRef]

31. Jing, L.; Ng, M.K.; Huang, J.Z. An Entropy Weighting K-Means Algorithm for Subspace Clustering of High-Dimensional Sparse Data. IEEE Trans. Knowl. Data Eng. 2007, 19, 1026-1041. [CrossRef]

32. Wang, T.C.; Lee, H.D. Developing A Fuzzy TOPSIS Approach Based on Subjective Weights and Objective Weights. Expert Syst. Appl. 2009, 36, 8980-8985. [CrossRef] 